increase the risk for developing AD. Results: The study population consisted of $183 \mathrm{MCI}$ patients at baseline. At follow-up, 74 patients were stable and 109 patients progressed to AD. The presence of significant depressive symptoms in MCI as measured by the CSDD (HR: 2.06; 95\% CI: 1.23 3.44; $\mathrm{p}=0.011$ ) and the GDS-30 (HR: $1.77 ; 95 \%$ CI: 1.10 - 2.85; $\mathrm{p}=0.025$ ) were associated with an increased the risk of progression to AD. The severity of depressive symptoms as measured by the GDS-30 was a predictor for progression too (HR: 1.06; 95\% CI: $1.01-1.11$; $\mathrm{p}=0.020$ ). Furthermore, also the severity of agitated behavior, especially verbal agitation, and the presence of purposeless activity were associated risk factors for progression, whereas diurnal rhythm disturbances in our study was associated with a decreased risk of progression. Conclusions: Depressive symptoms in MCI appear to be associated with an increased risk of progression to $\mathrm{AD}$.

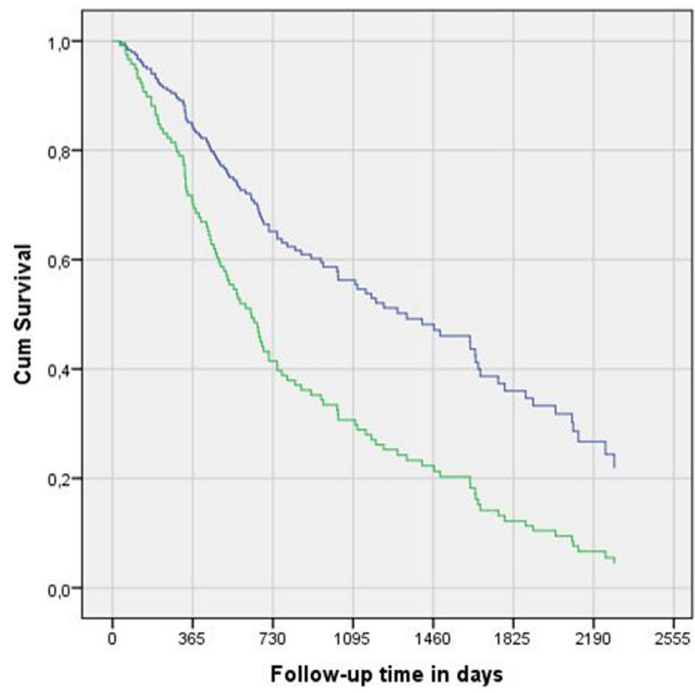

Depressive symptoms

$\neg 0$

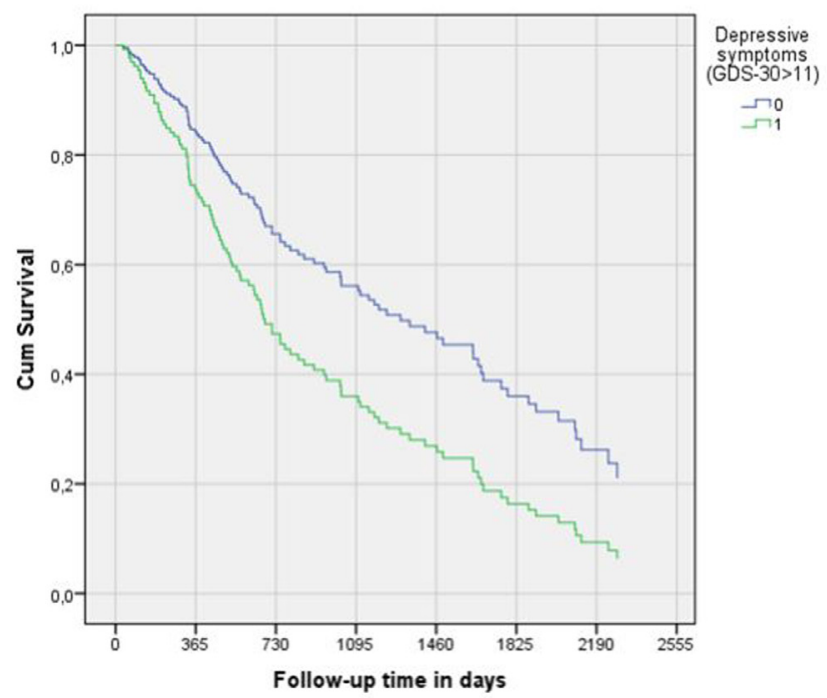

P3-155 THE DEM@CARE PROJECT SPEECH RECORDING AND AUTOMATIC ANALYSIS FOR THE ASSESSMENT OF ALZHEIMER DISEASE AND RELATED DISORDERS

Aharon Satt ${ }^{1}$, Alexandra König ${ }^{2}$, Alexander Sorin ${ }^{1}$, Orith Toledo-Ronen ${ }^{3}$, Ron Hoory ${ }^{3}$, Renaud David ${ }^{4}$, Frans R.J. Verhey ${ }^{5}$, Pauline Aalten ${ }^{6}$, Philippe H. Robert ${ }^{7},{ }^{1}$ Speech Technologies IBM Research Lab, Haifa,
Israel; ${ }^{2}$ School for Mental Health and Neuroscience, Maastricht University, Maastricht, Netherlands; ${ }^{3}$ IBM Speech Research Lab, Haifa, Israel; ${ }^{4}$ Nice Memory Clinic, Nice, France; ${ }^{5}$ Maastricht University, Maastricht, Netherlands; ${ }^{6}$ MUMC+, Maastricht, Netherlands; ${ }^{7}$ Hôpital de Cimiez, Lyon, France. Contact e-mail: a.konig@maastrichtuniversity.nl

Background: Assessment of early stage Alzheimer's disease (E-AD) and other dementia types, as well as Mild Cognitive Impairment (MCI) is complex; a broad range of heterogeneous assessment methods exist. Various types of dementia and MCI are manifested as irregularities in human speech and language, which have proven to be strong predictors for the disease presence and progression. Therefore, automatic speech analysis is expected to be a useful tool in providing indicators for assessment and detection of early stage dementia and MCI. Methods: 13 Healthy elderly subjects (HC), $22 \mathrm{MCI}$ patients and $23 \mathrm{E}-\mathrm{AD}$ patients were recorded while performing several short vocal cognitive tasks during a regular consultation. These tasks included verbal fluency, picture description and counting down. The voice recordings were processed in two steps: in the first step, vocal markers were extracted using speech signal processing techniques; in the second, the vocal markers were tested to assess their 'power' to distinguish between HC, $\mathrm{MCI}$ and E-AD. The second step included training automatic classifiers for detecting $\mathrm{MCI}$ and $\mathrm{E}-\mathrm{AD}$, based on machine learning methods, and testing the detection accuracy. Results: Preliminary results show the value of certain vocal tasks for distiguishing between $\mathrm{HC}$, MCI and E-AD. Using the above data, we demonstrated classification accuracy as follows: between HC and MCI: $82 \pm 8 \%$, between HC and E-AD: $87 \pm 5 \%$, and between MCI and E-AD: $81 \pm 7 \%$. Detailed description will be presented at the AAIC meeting. Conclusions: Decline in cognitive functioning affects speech production in different ways. Preliminary analysis indicates the potential value of vocal cognitive tasks for accurate automatic differentiation between $\mathrm{HC}$, $\mathrm{MCI}$ and E-AD. This can provide the clinician with meaningful information for assessment and early diagnosis purposes, based on non-invasive, simple and low-cost method. Investigations of new and improved vocal tasks, signal processing tools and pattern recognition tools, are planned.

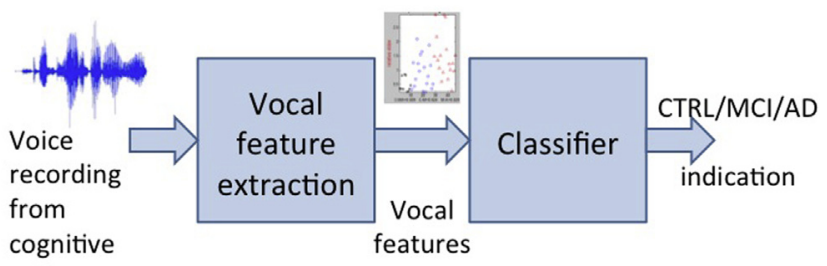

task

\section{P3-156 POOR RENAL FUNCTION IS ASSOCIATED WITH AMCI AT CROSS-SECTION: RESULTS FROM THE EINSTEIN AGING STUDY}

Andrea R. Zammit ${ }^{1}$, Mindy Joy Katz ${ }^{1}$, Markus Bitzer ${ }^{2}$, Jennifer Lai ${ }^{3}$, Richard B. Lipton ${ }^{1},{ }^{1}$ Albert Einstein College of Medicine, Bronx, New York, United States; ${ }^{2}$ University of Michigan, Ann Arbor, Michigan, United States; ${ }^{3}$ University of Michigan, Ann Arbor, Michigan, United States. Contacte-mail: andrea.zammit@einstein.yu.edu

Background: Though renal function is associated with cognitive impairment, few studies have examined the association between renal function and amnestic and non-amnsetic mild cognitive impairment (aMCI, naMCI). Further the prevalence of cognitive impairment seems to appear early in the course of renal disease, where i ndividuals with poor renal function experience greater decline in cognitive function. The objective of this study was to determine the association between eGFR and aMCI and naMCI and dementia in a relatively physically healthy community-dwelling sample. Methods: This a cross-sectional analysis conducted in the Einstein Aging Study (EAS). EAS enrolls community dwelling, English-speaking residents of Bronx county New York who are age 70+. Renal function was assessed using the estimated glomerular filtration rate (eGFR) using the CKD-EPI 
Table 1

Characteristics of the whole sample and according to eGFR (SDs, and CIs in brackets unless otherwise stated as \% for categorical variables).

\begin{tabular}{|c|c|c|c|c|c|}
\hline & Total & eGFR $<45$ & eGFR $45-59$ & $\mathrm{eGFR} \geq 60$ & \\
\hline eGFR, mean $( \pm S D)$ & $68.4(18.9,66.9-69.2)$ & $36.3(7.1,34.6-38.1)$ & $53.3(3.7,45.1-58.1)$ & $78.6(13.4,77.4-79.9)$ & \\
\hline Sex, males $(\%)$ & $253(39)$ & $28(11)$ & $45(18)$ & $180(71)$ & .031 \\
\hline Females $(\%)$ & $396(61)$ & $39(10)$ & $106(27)$ & $251(63)$ & \\
\hline Ethnicity (\%) & 649 & 67 & 151 & 431 & .035 \\
\hline Hispanic, White & 18 & 2 & 2 & 14 & \\
\hline Hispanic, Black & 7 & 1 & 3 & 3 & \\
\hline Asian & 5 & 0 & 0 & 5 & \\
\hline Other & 5 & 2 & 0 & 3 & \\
\hline Years in Education & $14.1(3.4,13.8-14.3)$ & $12.6(3.3,12.1-13.7)$ & $14.3(3.4,13.7-14.8)$ & $14.2(14.2,13.8-14.5)$ & .016 \\
\hline
\end{tabular}

Note. eGFR = estimated glomerular filtration rate. $\mathrm{SD}=$ standard deviation. $\mathrm{CI}=$ confidence interval.

Table 2

Odds ratio for aMCI, naMCI, and dementia predicted by eGFR group membership.

\begin{tabular}{|c|c|c|c|c|c|c|}
\hline & OR low eGFR vs high & \multicolumn{2}{|c|}{$95 \% \mathrm{CI}$} & OR moderate eGFR vs high & \multicolumn{2}{|c|}{$95 \% \mathrm{CI}$} \\
\hline $\mathrm{aMCI}$ & $2.23 *$ & 1.04 & 4.79 & 1.50 & 0.78 & 2.89 \\
\hline Dementia & 0.87 & 0.39 & 1.97 & 0.85 & 0.46 & 1.59 \\
\hline
\end{tabular}

Note. ${ }^{*} p<.05 \mathrm{OR}=$ odds ratio. $\mathrm{CI}=$ confidence interval. Low eGFR $=<45 \mathrm{~mL} / \mathrm{min} / 1.73 \mathrm{~m}^{2}$ moderate eGFR $=45-59 \mathrm{~mL} / \mathrm{min} / 1.73 \mathrm{~m}^{2}$ high eGFR $=$ $\geq 60 \mathrm{~mL} / \mathrm{min} / 1.73 \mathrm{~m}^{2}$ aMCI $=$ amnestic mild cognitive impairment. naMCI $=$ non amnestic mild cognitive impairment. Adjusted for age, sex, race and education.

equation. Categories of eGFR were predefined in units of $\mathrm{mL} / \mathrm{min} / 1.73 \mathrm{~m} 2$ as low $(<45)$, moderate $(45-59)$ and high $(\geq 60)$. We used multinomial logistic regressions to assess the relation of eGFR and 4 cognitive states: cognitive normality, aMCI, naMCI or dementia adjusting for age, sex, race, and education. Results: In 649 eligible participants mean age was $79.2( \pm \mathrm{SD}=5.6)$ and $61 \%$ were female. The sample included 496 (76.4\%) participants who were cognitively intact $42(6.5 \%)$ with aMCI, $38(5.9 \%)$ with naMCI, and $73(11.2 \%)$ with dementia at baseline. There were $67(10.3 \%)$ participants with low eGFR, 151 (23.3\%) with moderate eGFR and 431(66.4\%) with high eGFR. eGFR category was significantly and independently associated with aMCI but not naMCI or dementia. In comparison with the high eGFR group, participants in the low eGFR group had higher odds of having aMCI $(\mathrm{OR}=2.23, \mathrm{CI}=1.04-4.79)$ after adjusting for age, sex, race and education. Conclusions: In this cross-sectional analysis, low eGFR is associated with aMCI. Low eGFR could contribute directly to cognitive impairment through metabolic effects. Alternatively, aMCI and eGFR have shared risk factors that may contribute to this association. Longitudinal studies to clarify temporality and to control for a broader set of confounders and mediators are underway.

\section{P3-157 RELATIONSHIPS BETWEEN PERSONALITY CHARACTERISTICS AND CSF BIOMARKERS OF ALZHEIMER'S PATHOLOGY}

Deepti Kukreja ${ }^{1}$, Alessia Donati ${ }^{1}$, Laila Jaaidi ${ }^{1}$, Armin von Gunten ${ }^{2}$, Julius Popp ${ }^{3},{ }^{1}$ Centre Hospitalier Universitaire Vaudois, Lausanne, Switzerland; ${ }^{2}$ University Hospital of Lausanne, Lausanne, Switzerland;

${ }^{3}$ University Hospital Lausanne, Lausanne, Switzerland.

Contacte-mail: Deepti.Kukreja@chuv.ch
Background: In Alzheimer's disease (AD), the age of onset and the magnitude of cognitive impairment may depend on the individual capacity to compensate cerebral pathology and dysfunction (referred to as cognitive reserve). Beginning as early as in young adulthood, personality characteristics may contribute to cognitive reserve by their influence on lifestyle and health-related behavior. A resilient personality profile may be associated with lower risk of clinical dementia in persons with $\mathrm{AD}$ neuropathology.The aim of this ongoing study is to investigate whether premorbid and current personality characteristics modulate the relationships between cognitive performance and $\mathrm{AD}$ pathology as measured using CSF biomarkers at preclinical and early clinical disease stages. Methods: Fifty-one patients with mild cognitive impairment (MCI) defined as an intermediate clinical stage between normal cognition and dementia) and twenty-one older subjects with normal cognition were recruited so far. All of them completed assessments of premorbid and current personality characteristics by the NEO Personality Inventory Revised (as reported by a close family member) as well as of cognitive performance and functional status. Lumbar punctures were performed and the CSF concentrations of A $\beta 1-42$, total tau, and p-tau 181 were measured by ELISA. Multivariate regression analysis including possible confounders will be used to evaluate the associations of the cognitive test results with the personality domain scores, and the CSF markers for AD pathology. Results: Preliminary results show direct correlations of A $\beta$ 1-42 levels with scores of Extraversion and Conscientiousness in subjects with MCI. The effects of personality domain scores on the cognitive performance after controlling for the CSF markers will be reported. Conclusions: This study will contribute to better understand the impact of premorbid and current personality characteristics on the relationship between cerebral pathology and cognitive performance at very early clinical and preclinical stages of $\mathrm{AD}$. This approach may further help identify individuals at risk who may benefit from specific prevention programmes. 